\title{
Impact of Genetic Defects on Coronary Atherosclerosis among Turkish Cypriots
}

\author{
Cenk Conkbayir, $\mathbf{M D}^{1^{*}}$, Rezan Fahrioglu Yamaci, $\mathrm{PhD}^{2^{*}}$, Pinar Gencer, $\mathrm{MD}^{2}$, Burc Barin, $\mathrm{MSc}^{3}$, \\ Genco Yucel, $\mathrm{MD}^{4}$, Cenk Eray Yildiz, MD ${ }^{5}$, Murat Ugurlucan, $\mathrm{MD}^{6}$, A. Nazli Basak, $\mathrm{MD}^{2}$
}

${ }^{1}$ Department of Cardiology, Near East University, Nicosia, Cyprus; ${ }^{2}$ Department of Molecular Biology and Genetics, Bogazici University, NDAL, Istanbul, Turkey; ${ }^{3}$ Department of Biomedical Engineering, Near East University, Nicosia, Cyprus; ${ }^{4}$ Department of Cardiology, American Hospital, Istanbul, Turkey; ${ }^{5}$ Institute of Cardiology, Istanbul University, Istanbul, Turkey; ${ }^{6}$ Department of Cardiovascular Surgery, Istanbul University Istanbul Medical Faculty, Istanbul, Turkey

\section{ABSTRACT}

Background: The distribution of gene variants in the Turkish Cypriot population with coronary artery disease has not been investigated. In this study, we sought to research different genetic variants in the susceptibility to coronary artery disease and to identify possible associations between various clinical parameters and the genes involved in blood coagulation as well as glucose and lipid metabolism among the Turkish Cypriots and compared the results with the respective Turkish patients from Turkey.

Methods: A total of 187 individuals with coronary artery disease, namely 87 Turkish Cypriot individuals from Northern Cyprus, and 100 Turkish patients from Turkey, were investigated. The presence of CAD was documented with coronary angiography. The genetic susceptibility to coronary artery disease in the cohorts was studied using the variants FV Leiden (G1691A), Factor V R2 mutation (FVR2) (H1299R), PTH (G20210A), FXIII (V34L), $\beta$-Fibrinogen (-455 G>A), PAI-1 (4G/5G), HPA1 (a/b), MTHFR [C677T] and [A1298C], ACE (I/D), Apo B (R3500Q), and Apo E, in addition to the well-known risk factors associated with coronary artery disease.

Results: Age, male sex, diabetes mellitus, hyperlipidemia, triglycerides, HDL, and triglyceride/HDL ratio were significantly associated with $(P<.05)$; LDL $(P=.05)$ and total cholesterol $(P=.08)$ was marginally associated with coronary artery disease in the Turkish Cypriot population. The mutations in the MTHFR [C677T] gene variant were marginally higher in the Turkish Cypriot cohort when compared with

Received April 19, 2016; received in revised form fune 10, 2017; accepted fune 15, 2017.

* These authors contributed equally to the study.

Financial support for this study bas been provided by the Bogazici University.

Correspondence: Prof. A. Nazli Basak, Department of Molecular Biology and Genetics, Neurodegeneration Research Laboratory (NDAL), Bogazici University, 34342 Bebek, Istanbul / Turkey. 9021235966 79; fax: +90 2123597298 (e-mail: cenkconk@hotmail.com, muratugurlucan@yahoo.com. the Turkish patients from Turkey $(P=.06)$. No significant direct association of any of the gene variants with coronary artery disease in the Turkish Cypriot cohort could be defined. Several of the genetic variants were associated indirectly with the risk factors for coronary artery disease in Turkish Cypriots. MTHFR [A1298C] was found to be marginally associated with low HDL cholesterol $(P=.08)$. MTHFR [C677] wild-type allele was significantly associated with a decreased rate of high LDL cholesterol $(P<.05)$. The HPA-1 a/b variant was significantly associated with an increased rate of high total cholesterol levels $(P<.05)$.

Conclusion: Turkish Cypriot patients with coronary artery disease may be more affected by secondary factors, such as diabetes, hypertension, obesity, and sedentary life style when compared with genetic factors, which may be responsible for coronary artery disease.

\section{INTRODUCTION}

Coronary artery disease $(\mathrm{CAD})$ is the most common cause of death in Western countries [Wachira 2013]. The development and progression of CAD depends on risk factors, such as smoking, dietary fat consumption, high cholesterol, low-density lipoprotein (LDL) and triglyceride levels, highdensity lipoprotein (HDL) levels, physical inactivity, hypertension, diabetes, and genetic factors. CAD occurs in cases of angina pectoris and myocardial infarction (MI) due to atherosclerotic plaques that develop progressively. Epidemiological studies are consistent with an underlying multifactorial model of disease susceptibility with a significant polygenic component [Zdravkovic 2004; Peden 2011].

Multiple genes such as those involved in blood coagulation and the renin-angiotensin system act independently or synergistically to contribute to the development of CAD [Zintzaras 2008]. Genetic variants involved in these pathways have been intensively investigated for their involvement in the development and/or progression of CAD [Zintzaras 2008; Gardemann 1999; Russo 2001].

Genetic association analysis in a group of Turkish MI risk patients with diabetes mellitus showed that the MTHFR [A1298C] and PAI-1 deletion and ACE deletion variants were associated with cardiovascular events [Onrat 2012]. In another study, ACE deletion was the most frequent genetic 
variant in dyslipidemic patients [Kotaska 2012]. Although some studies revealed no association between ACE and CAD [Pfohl 1999], in a meta-study, a moderate association of the ACE I/D variant with CAD was established [Zintzaras 2008].

Although the distribution of gene variants in CAD patients was analyzed in different populations, the Turkish Cypriot population, which has a rate of CAD as high as that in other European countries, has not been investigated. In this study, 12 genetic variants were examined using univariate and multivariate logistic regression models of angiographically documented patients with CAD. The objectives of this study were as follows: 1) to investigate the genetic susceptibility to CAD of the Turkish Cypriot (TC) population from Turkey for the first time; 2) to compare the genetic susceptibility to CAD of TC and Turkish patient from Turkey (TR) cohorts; and 3) to identify possible associations between various clinical parameters and the genes involved in blood coagulation as well as glucose and lipid metabolism.

\section{METHODS}

\section{Study Populations}

In this study, a total of 187 individuals, namely $87 \mathrm{TC}$ individuals from Northern Cyprus and 100 TR individuals from Turkey, were investigated. The presence of CAD was documented by coronary angiography, which was applied due to clinical indications of ischemia, unstable angina pectoris, or myocardial infarction. Informed consent was obtained from the individuals involved in this study according to the local ethical procedures in accordance with the Helsinki Declaration of 1975 , revised in 2000 .

\section{Clinical Diagnosis}

The CAD was diagnosed using the Gensini score. Disease severity was assessed by the number of vessels involved (vessel score) and a severity score. Significant stenosis was determined visually and defined as $\geq 70 \%$ reduction in lumen diameter in any view compared with the nearest normal segment. A severity score was established for each case of coronary stenosis based on the degree of luminal narrowing and its topographic importance. A reduction in the lumen diameter and the X-ray appearance of concentric lesions and eccentric plaques were evaluated. Reductions of $1-25 \%, 26-50 \%, 51-75 \%, 76-90 \%$, and $91-99 \%$ and total occlusions were scored as 1, 2, 4, 8, 16, and 32, respectively. After the Gensini score was established, 36 points was determined as the cut-off value. Based on this value, patients were divided into two groups. Patients with a Gensini score $>36$ points were classified as having moderate to severe coronary atherosclerosis and diagnosed with CAD, and those with a Gensini score $\leq 36$ points were classified as having normal or mild coronary atherosclerosis and were used as controls.

\section{Genetic Analysis}

The blood samples were obtained from the study cohort with informed consent according to local legislations. The ViennaLab CVD Strip Assay was used to assess the following variants: FV Leiden (G1691A), FVR2 (H1299R),
PTH (G20210A), FXIII (V34L), $\beta$-fibrinogen (-455 G>A), PAI-1 (4G/5G), HPA1 (a/b), MTHFR [C677T], MTHFR [A1298C], ACE (I/D), Apo B (R3500Q), and Apo E.

DNA was extracted from blood samples using the standard phenol-chloroform protocol and subjected to the CVD Strip Assay (Vienna Lab, Austria) according to the manufacturer's recommendations. Briefly, two parallel multiplex PCRs were performed using 100-150 ng of DNA to amplify and biotin-label the 12 genetic variants, which were then denatured. Selective hybridization of the amplified products to a test strip containing allele-specific oligonucleotides was performed for $30 \mathrm{~min}$ at $45^{\circ} \mathrm{C}$ in a water bath. After removal of the unbound biotins via stringent washes, the PCR fragments were detected with a streptavidin-alkaline phosphatase conjugate and colored substrates. The genotype of an individual was established according to the manufacturer's recommendations.

\section{Statistical Methods}

Statistical analyses were performed using the SAS software, version 9.2 (SAS Institute, Cary, NC, USA). Comparisons of the baseline characteristics between the TC and TR cohorts were conducted using Fisher exact test (categorical factors) and Kruskal-Wallis test (continuous factors). Potential risk factors for CAD in the TC and TR cohorts were evaluated separately and in combined models. Twelve genetic variants were examined in univariate and multivariate logistic regression models as predictors of the following clinical factors: low (<35) HDL, high (>130) LDL, high (>200) total cholesterol, high $(>150)$ triglycerides, diabetes mellitus, and hypertension. Possible confounders, such as sex, age, and BMI category were also evaluated, and if they were significant predictors, they were adjusted for the multivariate models. In the TC cohort, the impact of daily dietary fat consumption, regular exercise, and diabetes mellitus (only in the high triglyceride model) were also evaluated. All factors with a $P$ value $<0.1$ in the univariate model were included in the initial multivariate

Table 1. Characteristics of the Entire Cohort $(n=187)$

\begin{tabular}{lccc}
\hline Clinical Characteristics & $\begin{array}{c}\text { Turkish Cypriots } \\
(\mathrm{N}=87)\end{array}$ & $\begin{array}{c}\text { Turkish Cohort } \\
(\mathrm{N}=100)\end{array}$ & $P$ \\
\hline Age, y (median [IQR]) & $63(57,69)$ & $50(42,58)$ & $<.0001$ \\
Male sex, n (\%) & $32(37)$ & $60(60)$ & .002 \\
Family history of CAD, n (\%) & $50(57)$ & $78(80)$ & .001 \\
Smoking history, n (\%) & $17(20)$ & $42(45)$ & .0004 \\
Diabetes, n (\%) & $24(28)$ & $13(14)$ & .03 \\
Hypertension, n (\%) & $49(56)$ & $36(37)$ & .01 \\
Hyperlipidemia, $\mathrm{n}(\%)$ & $29(36)$ & $48(53)$ & .03 \\
BMI (median [IQR]) & $29[25,31]$ & $27[24,32]$ & .08 \\
HDL level (median [IQR]) & $45[37,54]$ & $49[40,63]$ & .02 \\
\hline
\end{tabular}

IQR indicates interquartile range; $C A D$, coronary artery disease; BMI, body mass index; HDL, high-density lipoprotein. 
Table 2. Univariate Analysis Results and Characteristics of the Turkish Cypriot Cohort $(\mathrm{n}=87)$

\begin{tabular}{|c|c|c|c|}
\hline & $\mathrm{CAD}(\mathrm{n}=34)$ & No $C A D(n=53)$ & $P^{*}$ \\
\hline \multicolumn{4}{|l|}{ Clinical Characteristics } \\
\hline Age, y (median [IQR]) & $64[60,71]$ & $60[55,67]$ & 0.05 \\
\hline Male sex, n (\%) & $17(52)$ & $15(28)$ & 0.03 \\
\hline Family history of CAD, $\mathrm{n}(\%)$ & $19(56)$ & $31(58)$ & 0.81 \\
\hline Smoking history, $\mathrm{n}(\%)$ & $9(26)$ & $8(15)$ & 0.20 \\
\hline $\begin{array}{l}\text { Moderate/heavy alcohol con- } \\
\text { sumption, } \mathrm{n}(\%)\end{array}$ & $2(6)$ & $0(0)$ & $\mathrm{N} / \mathrm{A}$ \\
\hline Daily fruit consumption, n (\%) & $30(88)$ & $48(92)$ & 0.53 \\
\hline Daily meat consumption, $\mathrm{n}(\%)$ & $13(38)$ & $20(38)$ & 0.98 \\
\hline $\begin{array}{l}\text { Daily milk/yogurt consumption, } \\
\mathrm{n}(\%)\end{array}$ & $25(74)$ & $44(85)$ & 0.21 \\
\hline Daily dietary fat consumption, $\mathrm{n}(\%)$ & $19(56)$ & $19(37)$ & 0.08 \\
\hline Diabetes, n (\%) & $15(44)$ & $9(17)$ & 0.01 \\
\hline Hypertension, n (\%) & $22(65)$ & $27(51)$ & 0.21 \\
\hline Hyperlipidemia, n (\%) & $19(56)$ & $10(22)$ & 0.002 \\
\hline Regular exercise, $\mathrm{n}(\%)$ & $8(24)$ & $8(16)$ & 0.72 \\
\hline Regular check-up, n (\%) & $7(23)$ & $7(21)$ & 0.84 \\
\hline Body mass index (median [IQR]) & $28[24,31]$ & $30[26,32]$ & 0.05 \\
\hline Total cholesterol (median [IQR]) & $200[170,249]$ & 181 [167-212] & 0.03 \\
\hline Triglyceride level (median [IQR]) & $134[90,161]$ & $89[78,130]$ & 0.003 \\
\hline HDL level (median [IQR]) & $42[34,45]$ & $48[44,55]$ & 0.001 \\
\hline LDL level (median [IQR]) & $108[100,132]$ & $99[90,110]$ & 0.20 \\
\hline $\begin{array}{l}\text { Triglyceride/HDL ratio (median } \\
{[\mathrm{IQR}] \text { ) }}\end{array}$ & $3.2[2.3,4.4]$ & $1.8[1.6-2.5]$ & 0.001 \\
\hline $\begin{array}{l}\text { Blood glucose level (median } \\
{[[\mathrm{QR}] \text { ) }}\end{array}$ & $87[80,90]$ & $89[80,96]$ & 0.65 \\
\hline \multicolumn{4}{|l|}{ Genetic Characteristics } \\
\hline Factor V Leiden - no. (\%) & & & 0.43 \\
\hline WT & $28(82)$ & $48(91)$ & \\
\hline Het & $5(15)$ & $5(9)$ & \\
\hline Mut & $1(3)$ & $0(0)$ & \\
\hline FVR2- no. (\%) & & & 0.51 \\
\hline WT & $30(88)$ & $49(92)$ & \\
\hline Het & $4(12)$ & $4(8)$ & \\
\hline Mut & $0(0)$ & $0(0)$ & \\
\hline Prothrombin (PTH) - no. (\%) & & & $\mathrm{N} / \mathrm{A}$ \\
\hline WT & $34(100)$ & $49(92)$ & \\
\hline Het & $0(0)$ & $4(8)$ & \\
\hline Mut & $0(0)$ & $0(0)$ & \\
\hline Factor XIII - no. (\%) & & & 0.97 \\
\hline WT & $25(74)$ & $38(72)$ & \\
\hline Het & $8(24)$ & $13(25)$ & \\
\hline
\end{tabular}

Mut

$1(3)$

$2(4)$

$\beta$-fibrinogen - no. (\%)

WT
Het
Mut
PAl-1 - no. (\%)
$4 \mathrm{G} / 4 \mathrm{G}$
$4 \mathrm{G} / 5 \mathrm{G}$
$5 \mathrm{G} / 5 \mathrm{G}$

HPA-1 - no. (\%)

$$
a / a
$$

$\mathrm{a} / \mathrm{b}$

$\mathrm{b} / \mathrm{b}$

MTHFR C677T - no. (\%)

WT

Het

Mut

MTHFR A1298C - no. (\%)

$$
\text { WT }
$$

Het

Mut

HFE - no. (\%)

I/I

I/D

$D / D$

Apo B - no. (\%)

WT

Het

Mut

Apo E - no. (\%)
E2/2
E2/3
E2/4
E3/3
E3/4
E4/4

20 (59)

$12(35)$

19 (36)

2 (6)

5 (9)

9 (26) $\quad 12(23)$

$11(32) \quad 27(51)$

$14(41) \quad 14(26)$

$26(76) \quad 42(79)$

$7(21) \quad 10(19)$

$1(3) \quad 1(2)$

$12(35) \quad 19(36)$

$18(53) \quad 22(42)$

$4(12) \quad 12(23)$

$14(41) \quad 19(36)$

$18(53) \quad 27(51)$

$2(6) \quad 7(13)$

$5(15) \quad 8(15)$

$7(21) \quad 16(30)$

$0(0) \quad 0(0)$

$0(0) \quad 0(0)$
$29(55)$

0.21

0.93

0.59

$22(65) \quad 29(55)$

N/A

$34(100) \quad 53(100)$

0.68

*From the univariate logistic regression analysis.

In the univariate logistic regression analyses of the Turkish Cypriot cohort, the CAD cases had significantly higher total cholesterol levels, triglyceride levels, and triglyceride/HDL ratios; lower HDL levels; and higher proportions of males, diabetics, and hyperlipidemic individuals compared with the cases with no CAD. Of the CAD cases, the median BMI was marginally lower, the median age was marginally higher, and the daily dietary fat consumption was marginally higher when compared with the non-CAD cases. No significant difference was observed with respect to the genetic factors between the two groups. 
Table 3. Univariate Analyses and Characteristics of the Turkish Cohort $(n=100)$

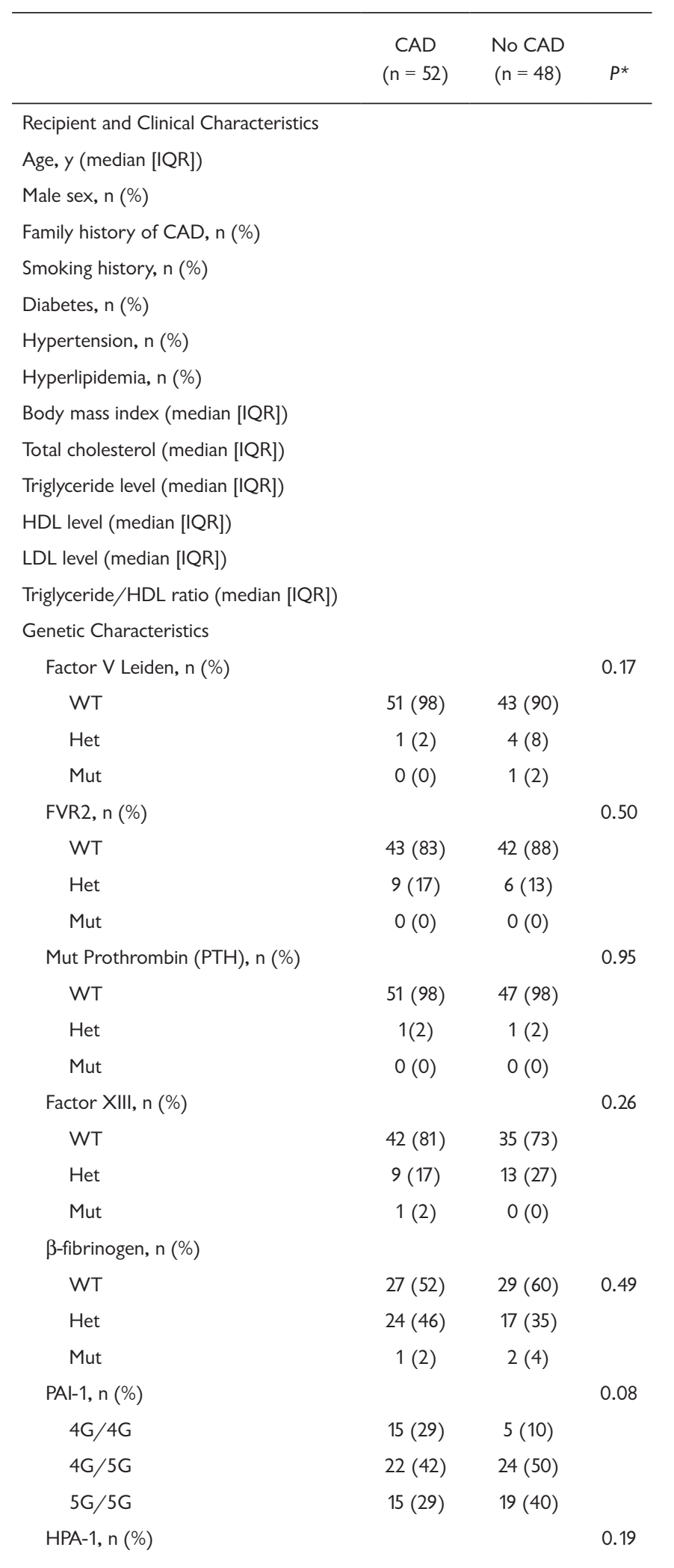

\begin{tabular}{|c|c|c|c|}
\hline$a / a$ & $40(77)$ & $41(85)$ & \\
\hline$a / b$ & $12(23)$ & $6(13)$ & \\
\hline$b / b$ & $0(0)$ & $1(2)$ & \\
\hline MTHFR C677T, n (\%) & & & 0.92 \\
\hline WT & $26(50)$ & $22(46)$ & \\
\hline Het & $22(42)$ & $22(46)$ & \\
\hline Mut & $4(8)$ & $4(8)$ & \\
\hline MTHFR A1298C, n (\%) & & & 0.08 \\
\hline WT & $16(31)$ & $25(52)$ & \\
\hline Het & $32(62)$ & $19(40)$ & \\
\hline Mut & $4(8)$ & $4(8)$ & \\
\hline HFE, n (\%) & & & 0.04 \\
\hline $1 / 1$ & $4(8)$ & $7(15)$ & \\
\hline I/D & $39(75)$ & $24(50)$ & \\
\hline$D / D$ & $9(17)$ & $17(35)$ & \\
\hline Apo B, n (\%) & & & $\mathrm{N} / \mathrm{A}$ \\
\hline WT & $52(100)$ & $48(100)$ & \\
\hline Het & $0(0)$ & $0(0)$ & \\
\hline Mut & $0(0)$ & $0(0)$ & \\
\hline Apo E, n (\%) & & & 0.94 \\
\hline $\mathrm{E} 2 / 2$ & $1(2)$ & $0(0)$ & \\
\hline $\mathrm{E} 2 / 3$ & $5(10)$ & $4(8)$ & \\
\hline$E 2 / 4$ & $1(2)$ & $0(0)$ & \\
\hline$E 3 / 3$ & $37(71)$ & $39(81)$ & \\
\hline $\mathrm{E} 3 / 4$ & $8(15)$ & $5(10)$ & \\
\hline $\mathrm{E} 4 / 4$ & $0(0)$ & $0(0)$ & \\
\hline
\end{tabular}

*From the univariate logistic regression analysis

In the univariate logistic regression analyses of the Turkish cohort, the CAD cases had a significantly higher age, BMI, triglyceride level, and triglyceride/ HDL ratio; a lower HDL level; and higher proportions of males and individuals with a smoking history, diabetes, hypertension, and hyperlipidemia (marginal) when compared with the cases with no CAD. Of the genetic factors, HFE classification was significantly associated with CAD, whereas PAI-1 and MTHFR A1298C were marginally associated with CAD.

CAD cases had an HFE distribution of $8 \% \mathrm{I} / \mathrm{I}, 75 \% \mathrm{I} / \mathrm{D}$, and $17 \% \mathrm{D} / \mathrm{D}$, whereas non-CAD cases had an HFE distribution of $15 \% \mathrm{I} / \mathrm{I}, 50 \% \mathrm{I} / \mathrm{D}$, and $35 \% \mathrm{D} / \mathrm{D}$.

CAD cases had a MTHFR A1298C distribution of 31\% WT, $62 \%$ Het, and $8 \%$ Mut, whereas the non-CAD cases had a MTHFR A1298C distribution of $52 \%$ WT, $40 \%$ Het, and $8 \%$ Mut.

CAD cases had a PAl-1 distribution of $29 \% 4 \mathrm{G} / 4 \mathrm{G}, 42 \% 4 \mathrm{G} / 5 \mathrm{G}$, and $29 \% 5 G / 5 G$, whereas the non-CAD cases had a PAl-1 distribution of $10 \%$ $4 \mathrm{G} / 4 \mathrm{G}, 50 \% 4 \mathrm{G} / 5 \mathrm{G}$, and $40 \% 5 \mathrm{G} / 5 \mathrm{G}$.

No other significant differences in genetic factors were observed between the two groups.

model. Subsequently, factors with a $P \geq 0.1$ were excluded, the model was re-fit, and all interactions were examined. A two-sided $P$ value <.05 was considered statistically significant. 
Table 4. Multivariate Logistic Regression Model of CAD in a Turkish Cypriot Cohort

\begin{tabular}{lcc}
\hline Multivariate Baseline Predictors & Odds Ratio $(95 \% \mathrm{Cl})$ & $P$ \\
\hline Triglyceride/HDL Ratio & $2.2(1.3,3.8)$ & .004 \\
BMI & $0.4(0.2,0.8)$ & .01 \\
Diabetes & $4.9(1.3,19.2)$ & .02 \\
Sex (Male) & $3.1(0.95,10.3)$ & .06 \\
\hline
\end{tabular}

$\mathrm{Cl}$ indicates confidence interval; CAD, coronary artery disease; BMI, body mass index; HDL, high-density lipoprotein.

Hyperlipidemia, total cholesterol, triglycerides, and HDL were not included in the multivariate model due to collinearity with the triglyceride/ $\mathrm{HDL}$ ratio.

\section{RESULTS}

In this study, a total of 187 individuals were clinically evaluated for the presence of CAD and the possible contribution of 12 gene variants in the development of this disease. Clinical assessment indicated the presence of CAD in 34/87 TC individuals and 52/100 TR individuals. Clinical parameters known to be involved in CAD, such as diabetes mellitus, hypertension, hyperlipidemia, total cholesterol, HDL, LDL, triglycerides, age, sex, BMI, and lifestyle (eg, smoking and eating habits) were also investigated for possible associations. The TC and TR cohorts were analyzed both separately and in combination.

\section{Association of Clinical Parameters with CAD}

In the combined analysis of the TC and TR cohorts, CAD was significantly associated with male sex, a higher triglyceride/HDL ratio, diabetes mellitus, and hypertension $(P<.05)$. However, subjects in the TC cohort had significantly higher age, marginally higher BMI, and significantly lower HDL when compared with the TR cohort (Table 1). The proportion of males and rates of family history of CAD, smoking, and hyperlipidemia were significantly higher in the TR cohort, whereas the rates of diabetes and hypertension were significantly higher in the TC cohort $(P<.05)$.

Potential risk factors for CAD in the TC cohort were evaluated as predictors of CAD in the univariate and multivariate logistic regression models. In the univariate model of the TC cohort, age, male sex, diabetes mellitus, hyperlipidemia, triglycerides, HDL, and the triglyceride/HDL ratio were significantly associated with CAD $(P<.05)$ (Table 2$)$. LDL and total cholesterol were marginally associated with CAD $(P=.05$ and $P=.08$, respectively). In contrast, the univariate analysis of the TR cohort indicated a significant association between CAD and male sex, smoking, diabetes mellitus, hypertension, BMI, increased triglycerides, HDL, and the triglyceride/HDL ratio $(P<.05)$ (Table 3$)$.

The multivariate analysis of the TC cohort showed that a high triglyceride/HDL ratio and diabetes mellitus were significantly associated with CAD $(P<.05)$. In addition, male sex was marginally associated with an increased rate of CAD in
Table 5. Multivariate Logistic Regression Model of CAD in the Combined Cohort

\begin{tabular}{lcc} 
Multivariate Baseline Predictors & Odds Ratio $(95 \% \mathrm{Cl})$ & $P$ \\
\hline Cohort (Turkish Cypriot) & $0.8(0.4,1.8)$ & .59 \\
Sex (Male) & $4.9(2.0,12.1)$ & .001 \\
Triglyceride/HDL Ratio & $1.5(1.1,1.9)$ & .004 \\
Diabetes & $4.2(1.5,11.3)$ & .005 \\
Hypertension & $2.5(1.03,6.2)$ & .04 \\
\hline
\end{tabular}

HDL indicates high-density lipoprotein.

Hyperlipidemia, triglycerides, and HDL were not included in the multivari-

ate model due to collinearity with the triglyceride/HDL ratio.

the multivariate model $(P=.06)$ (Table 4$)$.

Potential risk factors in the TR cohort were also evaluated as predictors of CAD in the univariate and multivariate logistic regression models (Table 5). In the univariate analysis, male sex, smoking, diabetes, hypertension, BMI, triglycerides, HDL, and the triglyceride/HDL ratio were significantly associated with CAD $(P<.05)$. However, in the multivariate model, a higher triglyceride/HDL ratio, diabetes mellitus, and male sex were significantly associated with a higher rate of CAD $(P<.05)$.

\section{Direct and Indirect Association of Genetic Factors with CAD}

The possible involvement of the genetic factors assessed was analyzed in both cohorts combined and separately. One major finding was the marginally higher rate of mutations in the MTHFR [C677T] gene variant in the TC cohort when compared with the TR cohort $(P=.06)$. However, there was no significant association of any of the gene variants with $\mathrm{CAD}$ in the TC cohort. In the univariate models for the TR cohort, a significant association of the I/D variant of the ACE gene $(P<.05)$, a marginal association of the heterozygous MTHFR [A1298C] variant $(P=.08)$, and an association of PAI-1 4G/4G $(P=.07)$ with CAD were observed. However, in the multivariate model, heterozygous MTHFR [A1298C] was significantly associated with a higher rate of CAD compared with the wild-type variant (odds ratio $[\mathrm{OR}]=5.2 ; 95 \%$ confidence interval $[\mathrm{CI}]: 1.3-21 ; P<.05)$.

Several of the genetic variants were associated with the clinical parameters of CAD rather than directly with CAD. From the multivariate analysis of the TC cohort, MTHFR [A1298C] was found to be marginally associated with low HDL cholesterol $(P=.08)$. Furthermore, the MTHFR [C677] wild-type allele was significantly associated with a decreased rate of high LDL cholesterol $(P<.05)$. The HPA-1 $\mathrm{a} / \mathrm{b}$ variant was significantly associated with an increased rate of high total cholesterol levels $(P<.05)$.

In the TR cohort, the multivariate analysis identified a significant association of heterozygous $\beta$-fibrinogen with an increased rate of high LDL $(P<.05)$. MTHFR [A1298C], which was marginally associated with high LDL values in the univariate model, lost its significance in the multivariate model. 
In the multivariate logistic regression model for the combined cohort, male sex, a higher triglyceride/HDL ratio, diabetes, and hypertension were the only factors that were significantly associated with a higher rate of CAD (Table 5).

\section{DIscussion}

In this study, several gene variants involved in CAD were analyzed for possible relationships in a group of TC patients for the first time in the literature. The analysis was then extended to a group of Turkish CAD patients. Each sample cohort was analyzed separately and then combined.

\section{Association of Clinical Parameters with CAD in a Group of Turkish Cypriot and Turkish Patients from Turkey}

Coronary artery disease is associated with many clinical factors, such as smoking, advanced age, male sex, diabetes mellitus, high systolic blood pressure, family history of CAD or MI, highfat diet, obesity, increased total and LDL cholesterol, increased triglycerides, and decreased HDL cholesterol in different populations [Var 2009]. Therefore, we assessed the possible correlation of CAD with any of these factors in a group of TC patients. Furthermore, the study was extended to a group of TR CAD patients, in whom some of the $\mathrm{CAD}$-associated clinical parameters were previously shown to be correlated with CAD [Ünal 2013].

In both the separate and combined cohorts, the common clinical parameters that were significantly associated with CAD were male sex, high triglyceride level, diabetes, and the triglyceride/HDL ratio. However, in the multivariate model, only diabetes and the triglyceride/HDL ratio remained significantly associated with CAD. Hyperlipidemia, triglycerides, and HDL were not included in the multivariate model due to colinearity with the triglyceride/HDL ratio. Other factors lost their significance in the multivariate model.

\section{Direct and Indirect Association of Genetic Factors with CAD}

Twelve variants of 10 different genes involved in coagulation were investigated for their possible association with $\mathrm{CAD}$ in a group of TC and patients from Turkey.

Although the association between the clinical parameters of CAD (high triglyceride/HDL ratio and diabetes, significantly, and male sex, marginally) and disease occurrence in the TC patients indicated correct sample selection, there was no association between any of the 12 genetic variants and CAD. Correlation studies in different populations have not always identified a positive relationship with one or more of the analyzed genetic factors. This pilot study included 34 mild or non-CAD and 53 CAD patients and may have been too small of a sample size for the analysis of a complex disease such as CAD. Therefore, increasing the sample size is essential to confirm the current findings in TC CAD patients.

Analysis of the TR cohort showed a significant association with the I/D variant of the ACE gene $(P<.05)$. The ACE gene has been intensively studied for its possible association with CAD in Turkish and other populations. However, the association of ACE with CAD remains under discussion because different studies have yielded contradicting results even in the same population. For example, our study confirmed previous findings in the Turkish population [Acarturk 2005; Isbir 1999; Akar 1998] but was not consistent with another study [Araz 2002] in which no association was found in type II diabetic CAD patients. Furthermore, a meta-analysis [Zintzaras 2008] indicated a moderate association of I/D with CAD. Therefore, a large sample size with a wide distribution from all regions of the country is essential to establish the possible association with ACE in the Turkish population. Furthermore, environmental factors should be critically analyzed because $\mathrm{CAD}$ is not caused only by genetic factors.

In addition to the ACE gene variant, the PAI-1 4G/4G variant was marginally associated with $\mathrm{CAD}(P=.07)$. This result contradicts several studies in which no significant association was established [Taymaz 2007; Agirbasli 2006].

Finally, heterozygous MTHFR [A1298C] was significantly associated with a higher rate of $\mathrm{CAD}(\mathrm{OR}=5.2 ; 95 \%$ CI: 1.3 $21 ; P=.02)$. In addition to associations between gene variants and $\mathrm{CAD}$, we have also observed an association of some gene variants with the known clinical parameters of CAD. In the TC cohort, both MTHFR variants were associated with LDL or HDL, such that [A1298C] was marginally associated with low HDL and [C677T] was significantly associated with high LDL. In the TC sample group, HDL and LDL were associated with CAD significantly and marginally, respectively. Previously, [C677T] was shown to be associated with low HDL [Lunegova 2011], indicating that the MTHFR gene may be related to the HDL level. However, the mechanism of this relationship remains elusive. Furthermore, in this cohort, the HPA-1 a/b variant was significantly associated with an increased rate of high total cholesterol levels. This is the first time that HPA-1 has been linked to cholesterol levels.

In the TR sample group, the MTHFR [A1298C] variant was marginally associated with high LDL levels. This finding also confirms the relationship of MTHFR with the lipoprotein level in the plasma. Finally, $\beta$-fibrinogen was significantly associated with an increased rate of high LDL in this sample group. The binding of lipoproteins to the cell surface is directly related to the circulating levels of plasminogen [von Depka 2000]; therefore, the association of high LDL with $\beta$-fibrinogen was confirmed in this study.

We also confirmed the significant relationship between the TG/HDL-C ratio and CAD risk in TC in a recent study by our group [Conkbayir 2015].

\section{Study Limitations}

The present study has several limitations. First, because of the relatively small sample size due to the single-center design, our findings may not be generalizable to the entire Cypriot population. Second, there is a large population size difference between the Turkish (nearly 80,000,000) and North Cyprus Turkish $(265,000)$ populations. Nevertheless, despite these limitations, because of the paucity of reliable information available on this genetic field and because Cyprus is an island nation and a significant portion of its population are foreign residents, the inclusion of Cyprus-born patients with Cypriot parents in our cohort represents a valuable contribution to the literature and provides genetic data on the prevalence of $\mathrm{CAD}$ in the TC population for the first time. 


\section{Conclusion}

In conclusion, this pilot study presents the first findings of the association of CAD with clinical and genetic parameters in a group of TC patients. However, to confirm the results, the study should be extended to a larger number of cohort. In this study, we confirmed the involvement of ACE in CAD disease and also presented new findings, such as the association of MTHFR [A1298C] with CAD in the Turkish cohort.

\section{ACKOWLEDGEMENT}

We gratefully acknowledge all volunteers who generously participated in this study.

\section{REFERENCES}

Acarturk E, Attila G, Bozkurt A, Akpinar O, Matyar S, Seydaoglu G. 2005. Insertion/deletion polymorphism of the angiotensin converting enzyme gene in coronary artery disease in southern Turkey. J Biochem Mol Biol 38:486-90.

Agirbasli D, Agirbasli M, Williams SM, Phillips JA 3rd. 2006. Interaction among 5,10 methylenetetrahydrofolate reductase, plasminogen activator inhibitor and endothelial nitric oxide synthase gene polymorphisms predicts the severity of coronary artery disease in Turkish patients. Coron Artery Dis 17:413-7.

Akar N, Aras O, Omürlü K, Cin S. 1998. Deletion polymorphism at the angiotensin-converting enzyme gene in Turkish patients with coronary artery disease. Scand J Clin Lab Invest 58:491-5.

Araz M, Aynacioglu S, Okan V, Akdemir I, Aktaran S. 2002. Angiotensin-converting enzyme gene polymorphism and coronary heart disease in Turkish type 2 diabetic patients. Acta Cardiol 57:265-9.

Conkbayir C, Ayça B, Ökçün EB. 2015. Lipid variables related to the extent and severity of coronary artery disease in non-diabetic Turkish Cypriots. Iran J Public Health 44:1196-203.

Gardemann A, Arsic T, Katz N, Tillmanns H, Hehrlein FW, Haberbosch W. 1999. The factor II G20210A and factor V G1691A gene transitions and coronary heart disease. Thromb Haemost 81: 208-13.

Isbir T, Yilmaz H, Ağaçhan B, Aydin M, Isbir CS. 1999. Association between angiotensin-converting enzyme gene polymorphism and coronary artery disease. IUBMB Life 48:205-7.

Kotaska K, Kolarova J, Kotrcova K, Cepova J, Prusa R. 2012. Correlation between common genetic variants and risk factors associated with prediction of cardiovascular diseases in dyslipidemic patients. Genet Test Mol Biomarkers 16:210-4.

Lunegova OS, Kerimkulova AS, Turdakmatov NB, et al. 2011. Association of C677T gene polymorphism of methylenetetrahydrofolate reductase with insulin resistance among Kirghizes. Kardiologiia 51:58-62.

Onrat ST, Akci O, Söylemez Z, Onrat E, Avşar A. 2012. Prevalence of myocardial infarction polymorphisms in Afyonkarahisar, Western Turkey. Mol Biol Rep 39: 9257-64.

Peden JF, Farrall M. 2011. Thirty-five common variants for coronary artery disease: the fruits of much collaborative labour. Hum Mol Genet 20:R198-205.

Pfohl M, Koch M, Prescod S, Haase KK, Häring HU, Karsch KR. 1999. Angiotensin I-converting enzyme gene polymorphism, coronary artery disease and myocardial infarction. An angiographically controlled study. Eur Heart J 20:1318-25.

Russo C, Girelli D, Olivieri O, et al. 2001. G20210A prothrombin gene polymorphism and prothrombin activity in subjects with or without angiographically documented coronary artery disease. Circulation, 103:2436-40.

Taymaz H, Erarslan S, Oner ET, Alkan T, Ağirbaşli M, Kirdar B. 2007. Sequence variations within the genes related to hemostatic imbalance and their impact on coronary artery disease in Turkish population. Thromb Res 119:55-62.

Ünal B, Sözmen K, Uçku R, et al. 2013. High prevalence of cardiovascular risk factors in a western urban Turkish population: a communitybased study. Anadolu Krdiyol Derg 1:9-17.

Var A, Utük O, Akçali S, Sanlidağ T, Uyanik BS, Dinç G. 2009. Impact of hemostatic gene single point mutations in patients with non-diabetic coronary artery disease. Mol Biol Rep 36:2235-43.

von Depka M, Nowak-Göttl U, Eisert R. 2000. Increased lipoprotein (a) levels as an independent risk factor for venous thromboembolism. Blood 96:3364-8

Wachira JK, Stys TP. 2013. Cardiovascular disease and bridging the diagnostic gap. S D Med 66:366-9.

Zdravkovic S, Wienke A, Pedersen NL, Marenberg ME, Yashin AI, de Faire U. 2004. Genetic influences on CHD-death and the impact of known risk factors: comparison of two frailty models. Behav Genet 34:585-92.

Zintzaras E, Raman G, Kitsios G, Lau J. 2008. Angiotensin-converting enzyme insertion/deletion gene polymorphic variant as a marker of coronary artery disease: a meta-analysis. Arch Intern Med 168:1077-89. 